\title{
Five miRNAs as Novel Diagnostic Biomarker Candidates for Primary Nasopharyngeal Carcinoma
}

\author{
Jin-Feng Tang ${ }^{1 \&}$, Zhong-Hua $\mathrm{Yu}^{2 \&}$, Tie Liu ${ }^{3}$, Zi-Ying Lin ${ }^{1}$, Ya-Hong Wang ${ }^{1}$, La- \\ Wei Yang ${ }^{1}$, Hui-Juan $\mathrm{He}^{1}$, Jun Cao ${ }^{4}$, Hai-Li Huang ${ }^{1}$, Gang Liu ${ }^{1 *}$
}

\begin{abstract}
MicroRNAs (miRNAs) play an essential role in the development and progression of nasopharyngeal carcinomas (NPC). Despite advances in the field of cancer molecular biology and biomarker discovery, the development of clinically validated biomarkers for primary NPC has remained elusive. In this study, we investigated the expression and clinical significance of miRNAs as novel primary NPC diagnostic biomarkers. We used an array containing 2 , 500 miRNAs to identify 22 significant miRNAs, and these candidate miRNAs were validated using 67 fresh NPC and 25 normal control tissues via quantitative real-time PCR (qRT-PCR). Expression and correlation analyses were performed with various statistical approaches, in addition to logistic regression and receiver operating characteristic curve analyses to evaluate diagnostic efficacy. qRT-PCR revealed five differentially expressed miRNAs (miR-93-5p, miR-135b-5p, miR-205-5p and miR-183-5p) in NPC tissue samples relative to control samples $(p<0.05)$, with miR-135b-5p and miR-205-5p being of significant diagnostic value $(p<0.01)$. Moreover, comparison of NPC patient clinicopathologic data revealed a negative correlation between miR-93-5p and miR183-5p expression levels and lymph node status $(p<0.05)$. These findings display an altered expression of many miRNAs in NPC tissues, thus providing information pertinent to pathophysiological and diagnostic research. Ultimately, miR-135b-5p and miR-205-5p may be implicated as novel NPC candidate biomarkers, while miR93-5p, miR-650 and miR-183-5p may find application as relevant clinical pathology and diagnostic candidate biomarkers.
\end{abstract}

Keywords: miRNAs - nasopharyngeal carcinomas - qRT-PCR - biomarkers

Asian Pac J Cancer Prev, 15 (18), 7575-7581

\section{Introduction}

Nasopharyngeal carcinoma (NPC) is highly prevalent in Southeast Asia relative to other regions, particularly in the Cantonese region around Guangdong in China (Qiu et al., 2011). Due to its unique location and a lack of specific symptoms, NPC is rarely detected during regular medical examinations and is often highly invasive in the late stages (Guo et al., 2013; Liu et al., 2013b). If these patients are diagnosed earlier or if relapses can be predicted sooner, clinical management would be greatly improved (Lin et al., 2014).

Despite key etiological factors including genetic susceptibility, environmental factors and latent infection with Epstein-Barr virus (EBV) being well established, the complex mechanisms driving NPC development and progression are not fully understood (Marquitz, 2012; Yang et al., 2013). One of the major events in NPC development is the inactivation of tumor suppressor genes, yet unlike other head and neck cancers, gene mutation or deletion is uncommon (Lee et al., 2002; Abbasi et al., 2011; Luo et al., 2012). Conversely, the down-regulation of tumor suppressor gene expression by microRNAs (miRNAs) is increasingly recognized to be an important mechanism of nasopharyngeal tumorigenesis ( $\mathrm{Li}$ et al., 2014; Ma et al., 2014). miRNAs are endogenous, small non-coding RNA molecules that completely or partially bind to target mRNAs and either block translation or lead to degradation to ultimately inhibit gene expression ( $\mathrm{Li}$ et al., 2011; Marquitz et al., 2012; Liu et al., 2014). miRNAs serve as essential posttranscriptional regulators that can specifically influence cancer development and progression, with aberrant expression patterns observed in multiple cancer types (Marquitz, 2012). Interestingly, miRNAs can act as either oncogenes or tumor suppressors, with both up- or down-regulation associated with carcinogenesis and cancer progression (Schetter, 2011). Furthermore, different cancer types, stages and differentiation grades may have unique miRNA expression signatures, thus enabling the use of miRNAs as biomarkers for cancer

${ }^{1}$ Clinical Research Center, Guangdong Medical College, ${ }^{2}$ Department of Oncology, ${ }^{4}$ Department of Pathology, Affiliated Hospital of Guangdong Medical College, Zhanjiang, ${ }^{3}$ Department of Hematology, the Second Affiliated Hospital, Medical School of Xi'an Jiaotong University, Xi'an, China ${ }^{\circledR}$ Equal contributors *For correspondence: gangliu11@gdmc.edu.cn 
detection and prognosis.

In order to assess the diagnostic significance of miRNAs in human NPC, we made miRNA profiling study about NPC tissues. We investigated the expression of miRNAs in NPC with microRNA Chip, and found that there were 22 miRNAs with significant changes. Further these miRNAs were validated by quantitative real-time PCR (qRT-PCR), and it was identified that there were five differentially expressed miRNAs in our study. Further analysis revealed that these five miRNAs might provide useful information pertaining to pathophysiological and diagnostic research.

\section{Materials and Methods}

\section{Clinical specimens}

Nasopharyngeal biopsy specimens were collected in the Department of Oncology at Affiliated Hospital of Guangdong Mecical College (Zhanjiang, China) from June 2012 to October 2013. The clinicopathological characteristics of 92 patients (including 67 NPC and 25 non-cancer patients, NC) were obtained in accordance with the Institutional Ethical Review Board of the Affiliated Hospital of Guangdong Medical College in China, with consent obtained from all patients. All relevant demographic and clinical pathology details were obtained for the 92 samples of patients, with samples collected prior to any therapeutic procedures such as chemotherapy or radiotherapy, using WHO histological classification of the NPC and the TNM classification of malignant tumors (Shanmugaratnam, 1991), as shown as Table 1. Individuals with no evidence of cancer were selected as non-cancer control subjects and were matched to patients based on age and gender. Tissue samples were snap frozen in liquid nitrogen upon collection and sent directly to the laboratory for RNA extraction.

\section{Immunohistochemical staining of tissue}

All biopsies immediately were fixed in $4 \%$ buffered paraformaldehyde, routinely processed, and embedded with paraffin. Serial $5 \mu \mathrm{m}$ histological sections were cut, mounted on glass slides coated with 3-aminopropyltriethoxysilane, and air-dried overnight at room temperature. The sections were then deparaffinised in xylene and rehydrated in ethanol. Haematoxylin and eosin ( $\mathrm{H}$ and $\mathrm{E})$ staining was performed in each specimen.

\section{Total mRNA isolation and miRNA array analysis}

Three freshly frozen NPC biopsy specimens and three normal nasopharyngeal epithelium specimens were sent to CapitalBio Corporation for noncoding RNA microarray analysis. The total RNA was extracted and purified using mirVana $^{\mathrm{TM}}$ miRNA Isolation Kit (Ambion, Austin, TX) following the manufacturer's instructions. The RNA integrity number (RIN) was assessed using an Agilent Bioanalyzer 2100 (Agilent Technologies, Santa Clara, CA), with samples with a $R I N \geq 6.0$ and $28 \mathrm{~S} / 18 \mathrm{~S} \geq 0.7$ used for the Agilent miRNA Chip. miRNA microarray profiles were obtained using the Agilent miRNA Microarray 19.0 containing 2047 miRNAs were comprehensive analysis of microRNA expression patterns in nasopharyngeal carcinoma and non-tumorous ( $3 \mathrm{NPC}$ and $3 \mathrm{NC}$ ). To identify differentially expresses miRNAs, the SAS system of microarray analysis was performed using a fold change value (FCV) of $\geq 2$ or $\leq 0.5$ and a signal value of $\geq 5$ to determine significance. For data analysis, inter-sample variance was normalized using quantile normalization strategies. Hierarchical clustering by Euclidean distance was used to cluster samples and groups with similar miRNA profiles. Differential analysis was performed using an unpaired t-test, ANOVA, and fold-change analysis. Differentially expressed miRNAs were selected based on t-test $(p \geq 0.001)$.

$R N A$ isolation, reverse transcription, and quantitative real-time PCR analysis

The 92 tissue samples (67 NPC and 25 non-cancer patients) were evaluated via qRT-PCR. Total RNA was extracted using TRIzol reagent (Invitrogen, Carlsbad, CA) and reverse transcribed using miRcute miRNA FirstStrand cDNA Synthesis Kit (Tiangen Biotech, China). miRNA expression was quantified via qRT-PCR using designed primers by Tiangen Biotech and the information of 22 miRNA is shown in Table 2. 22 miRNAs were examed via qRT-PCR with a miRcute miRNA qRT-PCR Detection Kit (Tiangen Biotech, China) and a Rotor-Gene Q2 plex HRM (Qiagen, Germany). All samples were normalized to endogenous controls and fold changes calculated relative to control. The control miRNA U6 was used for normalization, and relative expression levels were calculated using the $2^{-\Delta \Delta \mathrm{CT}}$ method (Liu et al., 2013a).

\section{Statistical analysis}

The SPSS17.0 program was used for general statistical analysis. The Wilcoxon test was used to examine the differences of miRNA expression among nasopharyngeal

Table 1. Clinical Characteristics of Nasopharyngeal Carcinoma Patients According to Training Set and Validation Set

\begin{tabular}{|c|c|c|c|c|}
\hline \multirow[t]{2}{*}{ Variable } & & \multicolumn{3}{|c|}{ Samples } \\
\hline & & \multicolumn{3}{|c|}{ NPC $(n=67) \quad$ Control $(n=25)$} \\
\hline \multirow[t]{2}{*}{ Sex } & Male & 56 & 16 & \\
\hline & Female & 11 & 9 & \\
\hline \multicolumn{2}{|c|}{ Average Ages (years) } & $51.87 \pm 2.257$ & $31.67 \pm 2.427$ & 0.0001 \\
\hline \multicolumn{2}{|c|}{ Histological types } & \multicolumn{3}{|c|}{ Squamous carcinoma } \\
\hline \multirow[t]{4}{*}{ T stages } & $\mathrm{T} 1$ & $12.50 \%$ & & \\
\hline & $\mathrm{T} 2$ & $28.10 \%$ & & \\
\hline & $\mathrm{T} 3$ & 18.8 & & \\
\hline & $\mathrm{T} 4$ & $40.60 \%$ & & \\
\hline \multirow[t]{4}{*}{$\mathrm{N}$ stages } & No & $19.40 \%$ & & \\
\hline & N1 & $30.60 \%$ & & \\
\hline & $\mathrm{N} 2$ & $33.30 \%$ & & \\
\hline & N3 & $16.70 \%$ & & \\
\hline \multirow[t]{4}{*}{ TNM stages } & I & $3.30 \%$ & & \\
\hline & II & $12.90 \%$ & & \\
\hline & III & $29.00 \%$ & & \\
\hline & IV & $54.80 \%$ & & \\
\hline \multirow[t]{2}{*}{ Family history } & yes & $16.10 \%$ & & \\
\hline & no & $83.90 \%$ & & \\
\hline \multirow[t]{2}{*}{ Smoking } & Smoking & $19.40 \%$ & & \\
\hline & No smoking & $80.60 \%$ & & \\
\hline \multirow[t]{2}{*}{ Region } & Zhanjiang & $26.80 \%$ & & \\
\hline & Other region & $70.20 \%$ & & \\
\hline
\end{tabular}

* (NPC vs Control) 


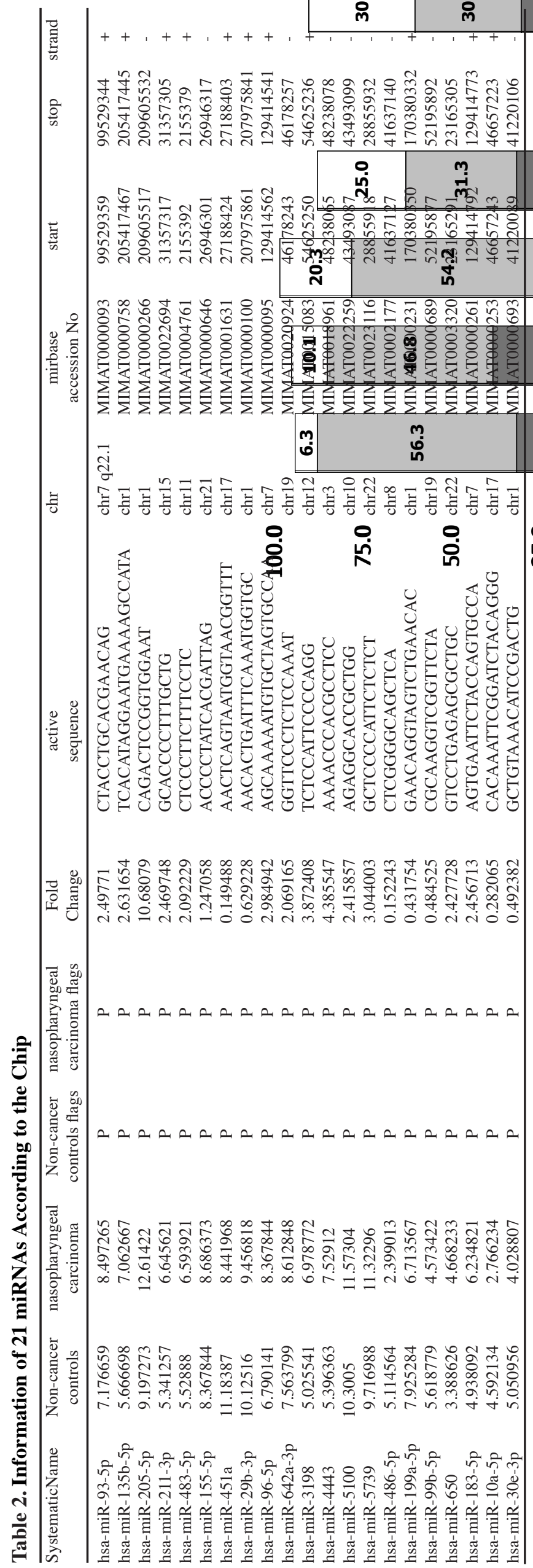

carcinoma and normal subjects. Spearman-Rho test was performed to compare the relative expression of miRNA in 67 NPC and 25 control tissues. The Kruskal Wallis test was applied to the examination of relationship between miRNA expression levels and clinicopathological characteristics. Receiver operating characteristic (ROC) curves and area under curves (AUC) were used to evaluate the prediction performance of the obtained. Specificity and sensitivity were determined based on sample class prediction: predicted values of the fitted Y for observations Experimental data were expressed as the mean \pm SEM. For comparison of means between two groups, a two-tailed t-test was used. Significance was accepted at $p<0.05$.

\section{Bioinformatics}

The selected miRNAs were further analyzed to identify their function and the pathways that they modulate using Ingenuity Pathway analysis (DAVID, http://david.abcc.ncifcrf.gov/home.jsp). This pathway analysis software identifies the putative targets of the input miRNA (s) and then develops networks and functions among the genes and targets. Before starting the analysis, the confidence was set to "highly predicted" and "experimental observed" and the species set to "human". The miRNA targets were then predicted using an integrated database including miRecords, Tarbase and TargetScan Human. The highly predicted targets were then matched and paired with mRNA expression data using the expression pairing function of miRWalk (http://www.umm.uniheidelberg.de/apps/zmf/mirwalk/). We assumed that the expression of a given miRNA was negatively correlated with the mRNA expression of its targets, which is a widely accepted and experimentally verified supposition (Li et al., 2011). The result identified the biological functions and canonical pathways associated with our data and were generated automatically using the core-analysis option in DAVID.

\section{Results}

\section{Histological stainings}

Two pathologists independently identified the results of nasopharyngeal carcinoma or chronic inflammation of nasopharyngeal mucosa for all samples. Representation $\mathrm{H}$ and $\mathrm{E}$ staining section is as shown in Figure 1.

Differentially expressed miRNA profiles between NPC and non-cancer patients identified using the Agilent human miRNA microarray 19.0

miRNAs were extracted from six samples with 3 NPC and 3 NC (non-cancer controls), independently. Of the 2047 miRNAs probed, 22 miRNAs were differentially expressed in NPC tissue relative to controls samples $(p<0.05, \mathrm{FCV} \geq 2$ fold or $\leq 0.5$ fold, signal value $\geq 5$ ) (Table 2), with 15 being up-regulated and 7 down-regulated in the NPC samples relative to the control. 
Jin-feng Tang et al

Validation of differentially expressed miRNAs using $q R T-P C R$

We next examined the expression of the 22 miRNAs in the 67 NPC and 25 normal control samples using qRTPCR. Following statistical analysis using a Wilcoxon test, five miRNAs (miR-93-5p $(p<0.01)$, miR-135b-5p $(p<0.001)$, miR-205-5p $(p<0.001)$, miR-650 $(p<0.05)$ and miR-183-5p $(p<0.05))$ were significantly overexpressed in NPC samples relative to the control samples. The expression of the other miRNAs was not significantly different between the NPC and normal control specimens $(p>0.05)$ (Figure 2).

The relationship between $\mathrm{miR}-93-5 p$ and $\mathrm{miR}-183-5 p$ expression and clinical histopathological features

Variations in the expression profiles of miR-93-5p, miR-135b-5p, miR-205-5p and miR-183-5p during different TNM stages, $\mathrm{T}$ stages and $\mathrm{N}$ stages were analyzed in NPC samples using the Kruskal-Wallis test (Figure 2). Our data revealed that miR-650 was up-regulated and related to the various $\mathrm{T}$ stages $(p<0.05)$, whereas miR93-5p and miR-183-5p were up-regulated and related to various $\mathrm{N}$ stages $(p<0.05$ or $p<0.01)$. However, the correlations between expression profiles of the five miRNAs and TNM stages were not significantly different $(p>0.05)$. Spearman-Rho analysis was performed to compare the relative expression of miR-93-5p and miR$183-5 p$ in NPC samples, with a significant correlation $(p<$ 0.05 ) noted with the $\mathrm{N}$ stages (miR-93-5p: $\mathrm{r}=-0.386$ and miR-183-5p: $r=-0.437$ ) as shown as Figure 3.

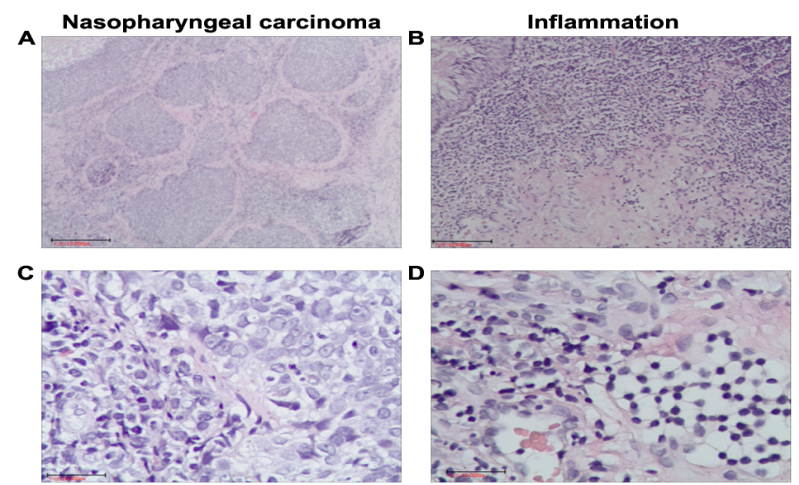

Figure 1. H\&E Staining Image of a Tissue Section Containing Nasopharyngeal Squamous Epithelium. (A and C). NPC in Tissue sections were stained with H\&E (original magnification, (A) (B)x200). (B and D). Typical pathology section in a chronic inflammation of nasopharyngeal mucosa patient in the study (original magnification, (B) (D) $\times 400$ )

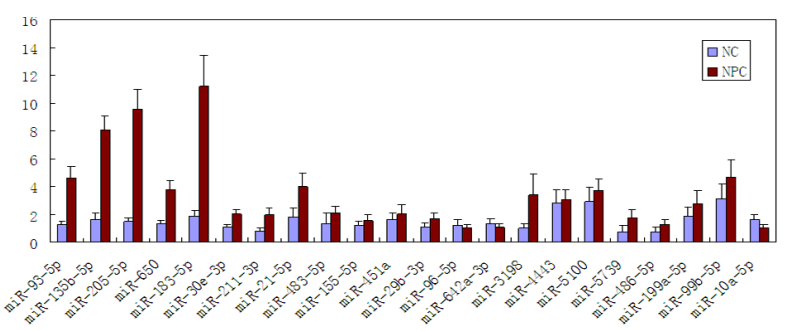

Figure 2. Relative Expression Levels of the 22 miRNAs in Nasopharyngeal Carcinoma and Normal Control Tissues. NS $p>0.05 ; * p<0.05 ; * * p<0.01 ; * * * p<0.001$
Assessing the predictive value of $m i R-205-5 p$ and $m i R$ $135 b-5 p$ expression for NPC detection

A Spearman-Rho test was performed to compare the relative expression of miR-93-5p, miR-135b-5p, miR-205$5 \mathrm{p}$, miR-650, and miR-183-5p in 92 tissues samples. The results revealed a significant correlation in miR-205-5p $(\mathrm{r}=0.521, p<0.01)$ and miR-135b-5p $(\mathrm{r}=0.489, p<0.01)$. To investigate the predictive value of the five different miRNAs in NPC, we measured their expression levels in $67 \mathrm{NPC}$ and $25 \mathrm{NC}$ tissue samples. The results of binary logistic regression analysis revealed a significant negative correlation between miR-205-5p and miR-135b$5 p$ expression and the risk of NPC $(p<0.05)$. Moreover, patients with high miR-135b-5p expression levels were at a higher risk of NPC compared with those with low miRNA expression profiles (odds ratio $(\mathrm{OR})=1.212$ ). Similarly, patients with high miR-205-5p expression levels were at a higher risk of NPC compared with those with low expression $(\mathrm{OR}=1.673)$. ROC curve analyses (Figure 4) also showed that both miRNAs could be used to differentiate NPC samples from control samples, with
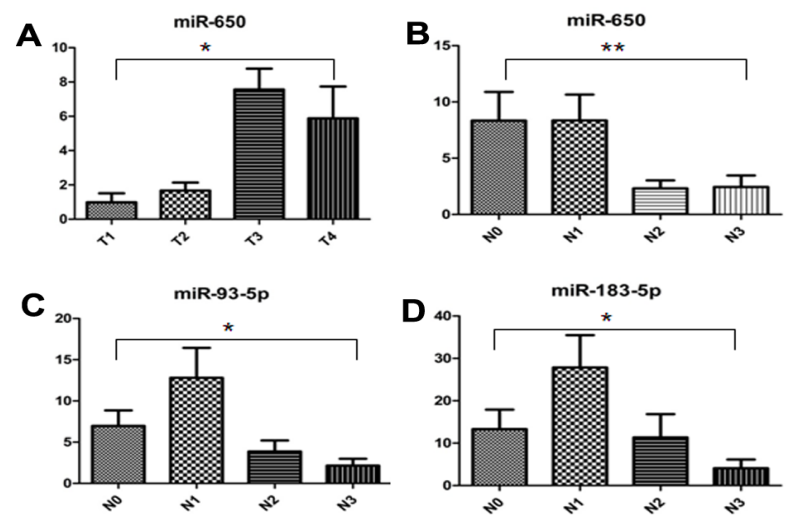

Figure 3. Relative Expression of miR-93-5p and miR183-5p in NPC T (a) and N stages (b, c, d). ${ }^{*} p<0.05$; $* * p<0.01$
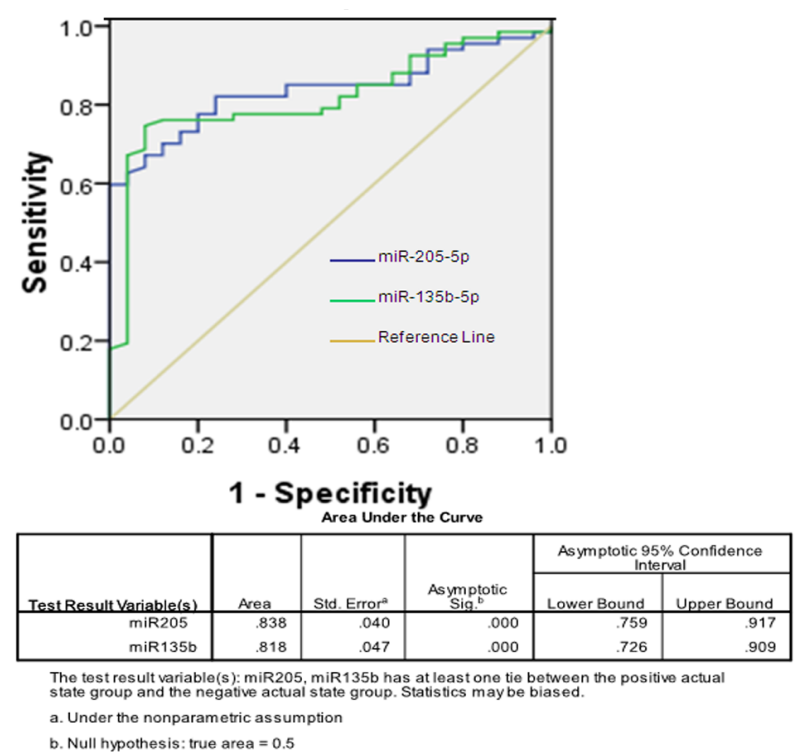

Figure 4. The Expression of miR-135b-5p and miR205-5p were Plotted to Discriminate Nasopharyngeal Carcinoma from Normal Control Patients. The area under the curve (AUC) was 0.818 and 0.838 
an area under the curve (AUC) of 0.818 for miR-135b-5p (95\%CI, 0.728-0.909) and 0.838 for miR-205-5p (95\%CI, $0.759-0.917)$ as shown as Figure 4.

\section{Discussion}

NPC is a malignancy with a high occurrence in Southern China. Accumulating evidence has demonstrated that miRNAs play important roles in various physiological and pathological processes, including carcinogenesis. miRNAs can function as oncogenes or tumor suppressors, with their dysregulation involved in multiple processes including cell proliferation, apoptosis, cell-cycle regulation and invasion in various diseases. For example, it was found miR-10b is up-regulated and promotes migration and invasion in metastatic NPC cell lines and associated with the expression of E-cadherin and MMP9 (Sun et al., 2013). It suggested that miRNAs work with adhesion molecules to influence NPC metastasis. In addition, miRNAs are potential novel biomarkers for various cancers. miRNA expression can be pathognomonic or tissue-specific, with human miRNAs easily preserved well in formalin-fixed and fresh snap frozen specimens ( $\mathrm{Lu}$ et al., 2005; Xi et al., 2007). Therefore, miRNA expression profiles are promising for characterizing tumors and could serve as potential diagnostic and prognostic markers to enhance treatment (Iorio and Croce. 2012). By performing an initial miRNA microarray followed by two sets of individual qRT-PCR analyses, we identified a correlation between two miRNAs and the N stages of NPC, and identified two miRNAs able to discriminate between NPC and normal tissues.

Among the five tissue miRNAs identified in this study, some were previously reported to play important roles in cancer (Zheng et al., 2013; Zhi et al., 2013). While NPC miRNA expression studies have highlighted the importance and potential roles of miRNAs in disease, a deeper understanding is needed (Liu et al., 2013b). miR-183-5p was reported to be an early predictive biomarker for prostate cancer with aggressive progression characteristics (Larne et al., 2013), while conversely in lung cancer its expression was decreased (Xie et al., 2013). The tumor suppressor PTEN (phosphatase and tensin homolog deleted on chromosome ten) is known to be regulated by miR-183-5p (Sarver et al., 2010). While no previous findings have characterized miR-183-5p in NPC, our data revealed a significant the correlation between miR-183-5p and the NPC N stages $(p<0.05)$. miR-93-5p has been found to be significantly up-regulated in laryngeal squamous cell carcinoma and in cisplatinresistant ovarian cancer cells, and may promote tumor growth and angiogenesis by targeting integrin- $\beta 8$ (Fu et al., 2012; Cao et al., 2013). Additionally, a decreased miR93 expression has been implicated as a prognostic factor for colon cancer (Xiao et al., 2013). In a colorectal cancer study, miR-93 suppressed the expression of ERBB2, p21 and the Vascular Endothelial Growth Factor VEGF, which all play roles in cell proliferation. Furthermore, miR-93 also suppressed colorectal tumor growth in tumor cell seeded Balb/c nude mice (Yang et al., 2012). miR-93 has also been reported to regulate VEGF expression in experimental models of diabetes both Asian Pac J Cancer Prev and Asian Pac J Cancer Prev (Long et al., 2010). While no previous findings have characterized miR-93-5p in NPC, our data revealed a significant correlation between miR-93-5p and the NPC N stages.

In NPC, host- and Epstein-Barr virus (EBV)-encoded miRNAs play key roles in almost all of the steps of epithelial cell carcinogenesis, including epithelialmesenchymal to stem-like transition, cell growth, migration, invasion and tumorigenesis (Chen et al., 2009; Liu et al., 2012; Lo et al., 2012). EBV is detected in all NPC cases regardless of differentiation subtype (Wolf et al., 1975; Raab-Traub et al., 1987). Further investigation of potential EBV miRNA target genes revealed the inhibition of tumor suppressor genes, such as PTEN, and the extensive dysregulation of several pathways that are commonly involved in NPC, such as Wnt signaling. In HeLa cells transfected with miR-BART6-5p RNAs, which is EBV-encoded, the expression of miR-205-5p was increased more than 2-fold (Iizasa et al., 2010). In the current study, miR-205-5p expression was considerably higher in NPC tissues relative to control samples. This finding is consistent with the understanding that EBV is detected in all NPC tumors and further supports the potential usefulness of miRNAs as diagnostic biomarkers.

Although few previous studies have quantified the expression of miR-205 in NPC patients using qRT-PCR, its expression has been assessed in lung cancer tissues. A recent study used high-throughput microarrays to measure miRNA expression in 122 adenocarcinoma and squamous cell carcinoma (SCC) specimens and demonstrated that the expression of hsa-miR-205 in malignant tissues is an accurate marker of SCC lung cancer (Lebanony et al., 2009). Furthermore, the relative expression of miR-205$5 p$ was significantly higher in non-small cell lung cancer tissues compared with non-cancer adjacent tissues paired specimens (Jiang et al., 2013). Consistent with these observations, our findings displayed an increased miR205-5p expression in NPC tissues. Nevertheless, the role of miR-205 remains controversial. For example, the downregulation of miR-205 is useful in distinguished melanoma from nevus (Kozubek et al., 2013). Moreover, the relative expression of miR-205-5p in any Gleason pattern was decreased significantly compared with normal tissues (Tsuchiyama et al., 2013), to include prostate cancer samples compared with non-prostate cancer samples (Larne et al., 2013). These results could be explained by a previous report that miRNA expression profiles are tissue-specific (Lu et al., 2005). In this study, we report for the first time that miR-135b-5p can serve as a biomarker capable of discriminate NPC from normal control patients. Although there are few studies describing the function of miR-135b-5p, a recent report showed that miR-135b-5p was upregulated in highly aggressive osteosarcoma cell lines (Lauvrak et al., 2013).

The current findings are preliminary and may be limited by the small number of patients examined. As such, further corroboration via large-scale and multicenter studies is necessary before clinically useful and reliable recommendations could be generated. Furthermore, future studies should be conducted over multiple time intervals 
to assess miRNA expression profiles during the pre- and post-operative periods, in addition to identify changes in these profiles over time.

These results are the first evidence suggesting that miR-135b-5p and miR-205-5p have the potential to be used as clinical markers for the diagnosis of NPC. Therefore, miR-135b-5p and miR-205-5p may have potential diagnostic applications in NPC patients. While additional studies are needed to confidently link these miRNA biomarkers with disease progression, survival and anti-cancer drug resistance development, they present themselves as potentially useful diagnostic tools in NPC.

\section{Acknowledgements}

We thank Dr. Yinghuan Xiong for the advice for statistical analysis on the manuscript. This project is supported by Science and Technology Innovation Fund of Guangdong Medical College (STIF201109) and by the Natural Science Foundation of China project (NSFC81172615), Guangdong Natural Science Foundation (S20122010008299), Science and Technology Planning Project of Guangdong Province (2012B031800223).

\section{References}

Abbasi A N, Zahid S, Bhurgri Y, et al (2011). Nasopharyngeal carcinoma - an update of treatment and acute radiation induced reactions from a tertiary-care hospital in Pakistan. Asian Pac J Cancer Prev, 12, 735-8.

Cao P, Zhou L, Zhang J, et al (2013). Comprehensive expression profiling of microRNAs in laryngeal squamous cell carcinoma. Head Neck, 35, 720-8.

Chen H C, Chen G H, Chen Y H, et al (2009). MicroRNA deregulation and pathway alterations in nasopharyngeal carcinoma. Br J Cancer, 100, 1002-11.

Fu X,Tian J,Zhang L, et al (2012). Involvement of microRNA-93, a new regulator of PTEN/Akt signaling pathway, in regulation of chemotherapeutic drug cisplatin chemosensitivity in ovarian cancer cells. FEBS Lett, 586, 1279-86.

Guo X-G, Xia Y (2013). The interleukin-18 promoter-607C> A polymorphism contributes to nasopharyngeal carcinoma Risk: Evidence from a Meta-analysis Including 1, 886 Subjects. Asian Pac J Cancer Prev, 14, 7577-81.

Iizasa H, Wulff BE, Alla NR, et al (2010). Editing of EpsteinBarr virus-encoded BART6 microRNAs controls their dicer targeting and consequently affects viral latency.J Biol Chem, $\mathbf{2 8 5}, 33358-70$.

Iorio MV, Croce CM (2012). microRNA involvement in human cancer. Carcinogenesis, 33, 1126-33.

Jiang M, Zhang P, Hu G, et al (2013). Relative expressions of miR-205-5p, miR-205-3p, and miR-21 in tissues and serum of non-small cell lung cancer patients. Mol Cell Biochem, 383, 67-75.

Kozubek J, Ma Z, Fleming E, et al (2013). In-depth characterization of microRNA transcriptome in melanoma. PLoS One, 8, 72699.

Larne O, Martens-Uzunova E, Hagman Z, et al (2013). miQ--a novel microRNA based diagnostic and prognostic tool for prostate cancer. Int J Cancer, 132, 2867-75.

Lauvrak S U, Munthe E, Kresse S H, et al (2013). Functional characterisation of osteosarcoma cell lines and identification of mRNAs and miRNAs associated with aggressive cancer phenotypes. Br J Cancer, 109, 2228-36.
Lebanony D, Benjamin H, Gilad S, et al (2009). Diagnostic assay based on hsa-miR-205 expression distinguishes squamous from nonsquamous non-small-cell lung carcinoma. J Clin Oncol, 27, 2030-7.

Lee N, Xia P, Quivey J M, et al (2002). Intensity-modulated radiotherapy in the treatment of nasopharyngeal carcinoma: an update of the UCSF experience. Int J Radiat Oncol Biol Phys, 53, 12-22.

Li MY, Liu JQ, Chen DP, et al (2014). Glycididazole sodium combined with radiochemotherapy for locally advanced nasopharyngeal carcinoma. Asian Pac J Cancer Prev, 15, 2641.

Li T, Chen J X, Fu X P, et al (2011). microRNA expression profiling of nasopharyngeal carcinoma. Oncol Rep, 25, 1353-63.

Lin JC, Chiang CF, Wang SW, et al (2014). Significance of Expression of Human METCAM/MUC18 in Nasopharyngeal Carcinomas and Metastatic Lesions. Asian Pac J Cancer Prev, 15, 245-52.

Liu N, Chen N Y, Cui R X, et al (2012). Prognostic value of a microRNA signature in nasopharyngeal carcinoma: a microRNA expression analysis. Lancet Oncol, 13, 633-641.

Liu N, Cui R X, Sun Y, et al (2014). A four-miRNA signature identified from genome-wide serum miRNA profiling predicts survival in patients with nasopharyngeal carcinoma. Int J Cancer, 134, 1359-68.

Liu N, Tang LL, Sun Y, et al (2013a). MiR-29c suppresses invasion and metastasis by targeting TIAM1 in nasopharyngeal carcinoma. Cancer Lett, 329, 181-8.

Liu X, Luo H N, Tian W D, et al (2013b). Diagnostic and prognostic value of plasma microRNA deregulation in nasopharyngeal carcinoma. Cancer Biol Ther, 14.

Lo A K, Dawson C W, Jin D Y, et al (2012). The pathological roles of BART miRNAs in nasopharyngeal carcinoma. $J$ Pathol, 227, 392-03.

Long J, Wang Y, Wang W, et al (2010). Identification of microRNA-93 as a novel regulator of vascular endothelial growth factor in hyperglycemic conditions. J Biological Chemis, 285, 23457-65.

Lu J, Getz G, Miska E A, et al (2005). MicroRNA expression profiles classify human cancers. Nature, 435, 834-8.

Luo W R, Gao F, Li S Y, et al (2012). Tumour budding and the expression of cancer stem cell marker aldehyde dehydrogenase 1 in nasopharyngeal carcinoma. Histopathology, 61, 1072-81.

Ma F, Zhang J, Zhong L, et al (2014). Upregulated microRNA301a in breast cancer promotes tumor metastasis by targeting PTEN and activating Wnt/beta-catenin signaling. Gene, 535, 191-7.

Marquitz A R, Raab-Traub N (2012). The role of miRNAs and EBV BARTs in NPC. Semin Cancer Biol, 22, 166-72.

Qiu J, Cosmopoulos K, Pegtel M, et al (2011). A novel persistence associated EBV miRNA expression profile is disrupted in neoplasia. PLoS Pathog, 7, 1002193.

Raab-Traub N, Flynn K, Pearson G, et al (1987). The differentiated form of nasopharyngeal carcinoma contains Epstein-Barr virus DNA. Int J Cancer, 39, 25-29.

Sarver A L, Li L, Subramanian S (2010). MicroRNA miR-183 functions as an oncogene by targeting the transcription factor EGR1 and promoting tumor cell migration. Cancer Res, 70, 9570-80.

Schetter A J, Harris C C (2011). Alterations of microRNAs contribute to colon carcinogenesis. Semin Oncol, 38, 734-72.

Shanmugaratnam K, 1991, Histological typing of tumours of the upper respiratory tract and ear. World Health Organization International Histological Classification of Tumours. Springer-Verlag, Berlin, p. 32- 34 pp. 
Sun XJ, Liu H, Zhang P, et al (2013). miR-10b promotes migration and invasion in nasopharyngeal carcinoma cells. Asian Pac J Cancer Prev, 14, 5533-37.

Tsuchiyama K, Ito H, Taga M, et al (2013). Expression of microRNAs associated with Gleason grading system in prostate cancer: miR-182-5p is a useful marker for high grade prostate cancer. Prostate, 73, 827-34.

Wolf H, Zur Hausen H, Klein G, et al (1975). Attempts to detect virus-specific DNA sequences in human tumors. III. EpsteinBarr viral DNA in non-lymphoid nasopharyngeal carcinoma cells. Med Microbiol Immunol, 161, 15-21.

Xi Y, Nakajima G, Gavin E, et al (2007). Systematic analysis of microRNA expression of RNA extracted from fresh frozen and formalin-fixed paraffin-embedded samples. RNA, 13, 1668-74.

Xiao Z G, Deng Z S, Zhang Y D, et al (2013). Clinical significance of microRNA-93 downregulation in human colon cancer. Eur J Gastroenterol Hepatol, 25, 296-01.

Xie L, Yang Z, Li G, et al (2013). Genome-wide identification of bone metastasis-related microRNAs in lung adenocarcinoma by high-throughput sequencing. PLoS One, 8, 61212.

Yang H J, Huang T J, Yang C F, et al (2013). Comprehensive profiling of Epstein-Barr virus-encoded miRNA species associated with specific latency types in tumor cells. Virol $J, \mathbf{1 0}, 314$.

Yang I P, Tsai H L, Hou M F, et al (2012). MicroRNA-93 inhibits tumor growth and early relapse of human colorectal cancer by affecting genes involved in the cell cycle.Carcinogenesis, 33, 1522-30.

Zheng G, Wang H, Zhang X, et al (2013). Identification and validation of reference genes for qPCR detection of serum microRNAs in colorectal adenocarcinoma patients. PLoS One, 8,83025 .

Zhi F, Cao X, Xie X, et al (2013). Identification of circulating MicroRNAs as potential biomarkers for detecting acute myeloid leukemia. PloS one, 8,56718 . 